\title{
MOLECULAR DOCKING STUDIES OF PHYTOCOMPOUNDS WITH TRANSCRIPTIONAL FACTORS IN HEPATOCELLULAR CARCINOMA
}

\author{
Sushma S. Murthy ${ }^{1, *}$ and T. Bala Narsaiah ${ }^{2}$ \\ ${ }^{1}$ Research Scholar, Jawaharlal Nehru Technological University Anantapur, AP, India \\ ${ }^{2}$ Department of Chemical Engineering, JNTUA College of Engineering, Anantapur, AP, India \\ *E-mail: sushmamicrobio@gmail.com
}

\begin{abstract}
Hepatocellular carcinoma is one of the majorly categorized forms of human malignancy and is the prime cause of cancer-related mortality. Transcription factors and co-factors play an important role in the cellular process performing as key regulators in the normal cell and in the cancerous cell. The present study investigates the potential interaction between the potent phytocompounds and key transcriptional factors involved in hepatocellular carcinoma (HCC) by using molecular docking studies. In the area of drug designing and development, computational modeling and simulation play a significant role. Screening of potent phytocompounds against targets by means of conventional methods is tedious and requires more time when compared to computational modeling and simulation studies. In the present study, the application of computational screening of phytocompounds against transcriptional factors was effectively used to determine their binding strength with the pythocompounds. Ten potential phytocompounds linalool, p-cymene, pelargonidin, harpagoside, 1, 8-cineole, afzelin, ginkgolide B, theophylline, bromelain and isoborneol and five transcription factors p53, AP-1, c-Myc, $\beta$-catenin and HIF-1 $\alpha$ were selected for docking studies. The 3D structures of both phytocompounds and transcription factors were obtained from Pubchem and PDB databases respectively. Docking studies were carried out by using AutoDockVina. On evaluating the docking results, it was found that phytocompounds Harpagoside, Bromelain and Afzelin showed strong binding affinity against their targets. These phytocompounds showed the binding free energy more than $6 \mathrm{kcal} / \mathrm{mol}$ and RMSD value between 4-7 $A^{\circ}$ with targets and can be potential ligands against the selected targets for further molecular investigations.
\end{abstract}

Keywords: Hepatocellular Carcinoma, Phytocompounds, Transcriptional Factors, Molecular Docking, AutoDockVina

@ RASĀYAN. All rights reserved

\section{INTRODUCTION}

Transcription factors have a direct role in decoding the information stored in DNA. These factors mainly are key regulators or master regulators that influence and controls the specific process and patterns of development in the specific cell. ${ }^{1}$ Transcription factor is also involved in controlling immune response pathways. $^{2}$ Differentiation of the cells are under the direct control of transcription factors which was proved in laboratory. ${ }^{3}$ Any kind of mutations in the transcription factors or their binding sites will lead to different types of human diseases., ${ }^{4,5}$

Hepatocellular carcinoma (HCC) is detected in more than half a million people each year and represents the fifth most common cause of tumor mortality worldwide. ${ }^{6}$ The mutation in the cellular level leads to uncontrolled proliferation of the cell, where the cell replicates at higher rate and ends in multiple growth causing inhibition of apoptosis. ${ }^{7}$ Transcriptional factors plays a very vital role in regulating the cellular process in normal cell and in transformed cell i.e. malignant cell, as these key factors are involved in integrating and converting in extracellular and intracellular signaling mechanisms and hence acts as central regulators for various molecular mechanisms. It is reported that $10 \%$ of the human gene transcribes and translates into DNA-binding proteins. Most of the DNA binding proteins are involved in

Rasayan J. Chem., 12(4), 2030-2038(2019)

http://dx.doi.org/10.31788/RJC.2019.1245475

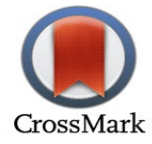


transcriptional machinery by acting as transcription factors and co-factors. ${ }^{8}$ These transcriptional factors play a very important role in molecular mechanisms in a cell for the progression of normal cell to malignant cells. In tumor cells, activation or overexpression; inactivation or dysregulation of these factors or proteins will be observed when compared to a normal cell which represents the last step in signaling pathways that affects the normal process of a cell to convert to an oncogenic manner by involving in various molecular mechanisms like proliferation, differentiation, apoptosis, senescence and migration. ${ }^{9,10}$ In this study, the key transcriptional factors selected are p53, AP-1, c-Myc, $\beta$-catenin and HIF-1 $\alpha$.

These transcriptional factors in human hepatocarcinogenesis are transcriptionally dysregulated. c-Myc is a regulatory gene that codes the transcriptional factors responsible for proliferation, apoptosis and differentiation. ${ }^{11,12} \beta$-catenin is an important structural protein which acts as a regulatory molecule in cadherin-mediated cell to cell adhesion $^{13}$, and has vital role tissue generation and differentiation. ${ }^{14}$ Transcription factor AP-1 is usually seen in multiple signaling pathways at the receiving end, ${ }^{15}$ in which transcription factor (c-Jun) is involved in various cellular processes which includes cell proliferation, survival and transformation of cell. The study has reported the expression of c-Jun protein in various tissues and is a potent oncogene for different cell types and is especially significant for hepatocytes $^{16}$. p53 gene is frequently subjected to mutations which makes it important to study its role in human cancer. p53 is not only a tumor suppressor gene but also controls various physiological processes in the cell due to its function as a transcriptional regulator, regulating downstream targeted genes. ${ }^{17}$ Upon DNA damage p53 also plays a role in arresting G1 cell cycle. Its function in cell death and apoptosis has been validated. ${ }^{18} \mathrm{HIF}-1 \alpha$ is a crucial transcription factor that contributes to the tumor EMT (epithelial-mesenchymal transition) which is critical for vasculogenic mimicry (VM). VM and EMT describe elevated risk of metastasis and tumor recurrence. ${ }^{19}$ In order to investigate the potency of phytocompounds (linalool, p-cymene, pelargonidin, harpagoside, 1, 8-cineole, afzelin, ginkgolide B, theophylline, bromelain and isoborneol) as transcriptional inhibitors which could be used in the hepatocellular carcinoma therapy, molecular docking studies were performed through Insilico approaches. This can be used as a tool for discovering a novel drug, which acts as lead molecule and can be screened from large pools of drugs and subjected to in-vitro analysis.

\section{EXPERIMENTAL}

\section{Identification and Retrieval of 3D Structure of Phytocompounds and Transcriptional Factors}

The 3D structures of 10 phytocompounds were retrieved from pubchem compound database at NCBI server and KEGG Drugs databases and 3D structures of transcriptional factors obtained from PDB databas $^{20}$. The compound with a similarity threshold over $90 \%$ according to the Tanimoto's coefficients ${ }^{21}$ and Lipinski rule ${ }^{22}$, were retrieved to understand the structural information for the modeling of phytocompounds. 3D structures of selected transcriptional factors were later preprocessed before their usage in Docking.

\section{Energy Minimization}

Before using 3D structures of phytocompounds, they were subjected to energy minimization using Avogadro software ${ }^{23}$ until stable atomic conformation of each phytocompound obtained. During energy minimization, geometry of atoms is rearranged to get stable energetically favorable structure. Other than energy minimization, Avogadro software helps in building chemical structures, optimization of structures, analyzing molecule, visualizing molecule, calculating quantum mechanics and calculation of electron density.

\section{Protein Preparation and Active Site Identification}

The X-ray crystallographic structure of p53 (PDB ID: 5G4N) with resolution 1.35A ${ }^{0}$, AP-1 (PDB ID: $1 J U N$ ) with high resolution, c-Myc (PDB ID: 4Y7R) with resolution $1.90 \mathrm{~A}^{0}, \beta$-catenin (PDB ID: $1 \mathrm{P} 22$ ) with resolution $2.95 \mathrm{~A}^{0}$ and HIF-1 $\alpha$ (PDB ID: 5LA9) with resolution $2.81 \mathrm{~A}^{0}$ were downloaded from protein data bank (PDB). ${ }^{24}$ Some of these structures were in complex form; these structures were prebound with ligands. Before docking, these structures were processed by removing prebound ligands 
and after removing prebound ligands along with water molecule, deficient hydrogen atoms were added at an appropriate position within the target. After preprocessing of target, Kollman charges were added to every atom of the protein and degree of the freedom is defined for the rotatable bond while using AutoDockTools-1.5.6. ${ }^{25}$ Grid box was generated within every preprocessed target using Auto grid tool. The dimension of grid box was varied according to transcription factor and ligand, but in most the cases it was kept within $10-20 \mathrm{~A}^{0}$. The average dimension of the grid box was $\left(40 \mathrm{~A}^{0} \mathrm{X} 40 \mathrm{~A}^{0} \mathrm{X} 40 \mathrm{~A}^{0}\right)$, it was varied slightly with grid spacing $0.375 \mathrm{~A}^{0}$. The grid box has played a very crucial role in the recognition of active or binding sites within protein targets while performing molecular docking studies. With these changes, targets were used in the process of molecular docking to study molecular interactions between phytocompounds and targets.

\section{Molecular Docking Studies}

The site for binding in five proteins was predicted by the protocol of ligand-receptor interaction. For each and every binding site in protein, the docking of all ten ligands was done by using AutoDockTools1.5.6. ${ }^{26}$ After docking all the phytocompounds with each target, docking results were tabulated, three compounds depending on the cut-off values of Gibbs free energy $(\Delta \mathrm{G})$ which is between -88 to -6.0 $\mathrm{kcal} / \mathrm{mol}$ and RMSD within 4-8 $\mathrm{A}^{0}$ were selected for further analysis. One of the important tools in computational modeling for designing drug based on its structure is Automated docking, AutoDockVina is software for molecular docking studies and for virtual screening and by procedures of multiple linear regression fitting, two scoring functional components is derived which is in a linear combination. The scoring function of Vinardo (VinaRaDii Optimized) was used in AutoDockVina. Vinardo uses Gaussian attraction in combination with quadratic repulsion. Molecular Docking interactions between transcriptional factors and docked compounds were analyzed using Discovery Studio 3.5. Based on docking score, good confirmation of each ligand was retrieved and analyzed further. ${ }^{27}$

\section{RESULTS AND DISCUSSION}

The systematic and scientific way of small molecules docking against the active receptor site is an important tool for the discovery of novel and potent drugs. ${ }^{28}$ The best confirmation binding site of the target molecule with its receptor is decided on its binding affinity in terms of their binding energies or their interaction energies in particular confirmation is considered as the best docking confirmation. After preprocessing of transcription factors, the binding pocket was identified and analyzed for the presence of pharmacophore. The pharmacophore within the binding pocket of p53 and $\beta$-catenin are having approximately 5-6 amino acids and pharmacophore of both the transcription factors are rich with hydrogen donors and hydrogen acceptors. Phytocompounds were successfully docked to binding pocket of both the transcription factors and interaction was depicted in Fig.-1 and Fig.-2 respectively. Similarly each of the phytocompound was docked into binding pocket of every transcription factor. During molecular docking, the docking software-generated 10 most ideal conformers for every ligand. Upon successful docking of all conformers to the binding pocket of receptor, all the conformers were ranked in a log file based on their binding free energy and RMSD values. The best-docked pose within binding cavity was selected and saved in separate pdb file. Based on binding free energy and RMSD, it was found that harpagoside, bromelain and afzelin were ideal ligands for all selected transcription factors (AP-1, $\beta$-catenin, p53, c-Myc and HIF-1 $\alpha$.) (Table-2).

Table-1: Binding Affinity of Phytocmpounds with Crystal Structure and Dynamically Stable Conformation of Transcriptional Factors.

\begin{tabular}{l|l|l|l|l|l|l}
\hline $\begin{array}{l}\text { Lead } \\
\text { Compound }\end{array}$ & Pubchem id & $\begin{array}{l}\Delta \mathrm{G} \\
\mathrm{p} 53 \\
(\mathrm{kcal} / \mathrm{mol})\end{array}$ & $\begin{array}{l}\Delta \mathrm{G} \\
\text { AP-1 } \\
(\mathrm{kcal} / \mathrm{mol})\end{array}$ & $\begin{array}{l}\Delta \mathrm{G} \\
\mathrm{C}-\mathrm{Myc} \\
(\mathrm{kcal} / \mathrm{mol})\end{array}$ & $\begin{array}{l}\Delta \mathrm{G} \\
\beta \text {-catenin } \\
(\mathrm{kcal} / \mathrm{mol})\end{array}$ & $\begin{array}{l}\Delta \mathrm{G} \mathrm{HIF-} \\
1 \alpha . \\
(\mathrm{kcal} / \mathrm{mol})\end{array}$ \\
\hline Linalool, & CID_6549 & -3.4 & -5.7 & -3.9 & -4.1 & -3.6 \\
\hline p-cymene & CID_7463 & -3.5 & -3.9 & -4.8 & -4.9 & -4.0 \\
\hline Pelargonidin & CID_440832 & -5.8 & -5.2 & -7.0 & -6.8 & -6.2 \\
\hline
\end{tabular}


RASĀYAN J. Chem.

Vol. 12 | No. 4 |2030 - 2038| October - December | 2019

\begin{tabular}{l|l|l|l|l|l|l}
\hline Harpagoside & CID_5281542 & -6.6 & -6.0 & -7.2 & -8.1 & -5.8 \\
\hline 1, 8-cineole & CID_2758 & -3.8 & -3.7 & -4.8 & -4.7 & -3.9 \\
\hline Afzelin, & CID_5316673 & -6.3 & -5.7 & -6.5 & -7.9 & -6.3 \\
\hline Ginkgolide & CID_6324617 & -6.0 & -5.7 & -6.1 & -8.1 & -6.0 \\
\hline Theophylline & CID_2153 & -4.4 & -3.6 & -4.9 & -4.7 & -4.2 \\
\hline Bromelain & CID_44263865 & -7.0 & -4.3 & -7.0 & -8.8 & -5.8 \\
\hline Isoborneol & CID_6321405 & -4.4 & -3.7 & -4.6 & -5.1 & -4.2 \\
\hline
\end{tabular}

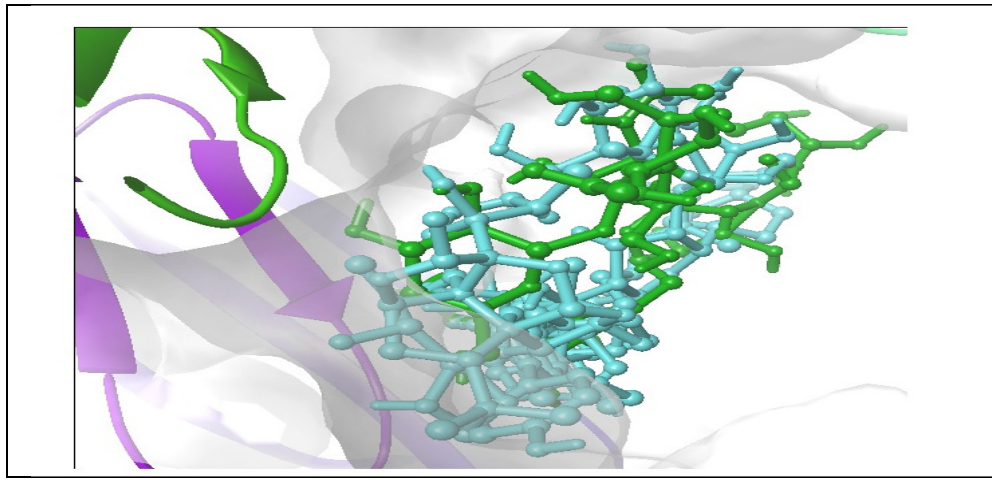

Fig.-1: Superposition of the Docking Poses of Ten Phytocompounds in Active Site of Dynamically Stable Structure of B-Catenin. The Structure of all Phytocompounds is Represented in The Blue Color and the High Affinity

Compound Bromelain Represented in the Green Color.

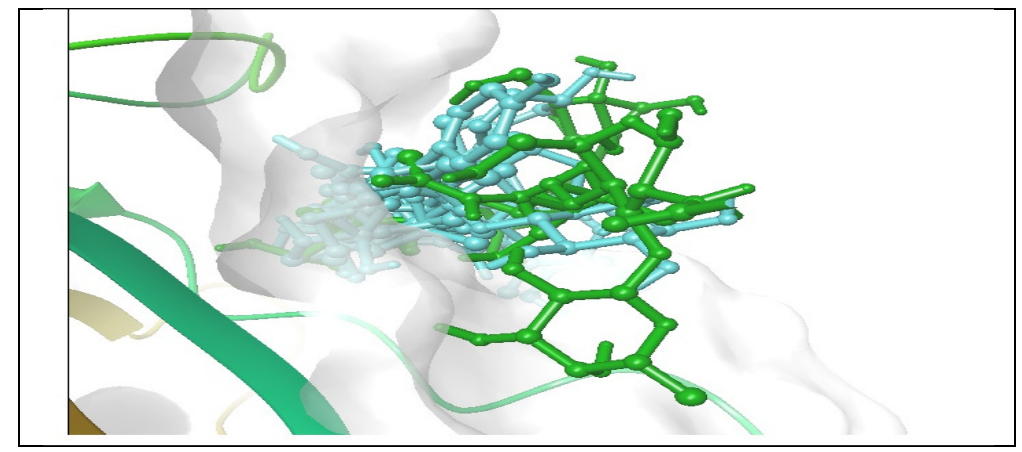

Fig.-2: Superposition of the Docking Poses of Ten Phytocompounds in Active Site of Dynamically Stable Structure of P53. The Structure of all Phytocompounds is Represented in the Blue Color and The High Affinity Compound Bromelain Represented in the Green Color.

Table-2: High Affinity Docking Compounds

\begin{tabular}{|c|c|c|c|c|}
\hline Lead Compound & Protein & Affinity & $\begin{array}{l}\text { Interacting } \\
\text { Residues }\end{array}$ & Distance $\left(\mathrm{A}^{0}\right)$ \\
\hline \multirow[t]{2}{*}{ Bromelain } & $\beta$-catenin, & -8.8 & $\begin{array}{l}\text { Tyr275(1HB) } \\
\text { Arg354(1HB) } \\
\text { Asp316(1HB) } \\
\text { Gln526(1HB) } \\
\text { Arg477(2HB) } \\
\text { Gln274(1HB) }\end{array}$ & $\begin{array}{l}1.99 \\
2.66 \\
2.78 \\
2.24 \\
2.13 \& 2.14 \\
2.23\end{array}$ \\
\hline & p53 & -7.0 & $\begin{array}{l}\text { Glu224(1HB) } \\
\text { Ser227(1HB) } \\
\text { His233(1HB) } \\
\text { HOH2196(2HB) }\end{array}$ & $\begin{array}{l}2.08 \\
1.85 \\
2.67 \\
2.29 \& \\
2.30\end{array}$ \\
\hline
\end{tabular}


RASĀYAN J. Chem.

Vol. 12 | No. 4 |2030 - 2038| October - December | 2019

\begin{tabular}{l|l|l|l|l}
\hline Harpagoside & AP-1 & -6.0 & $\begin{array}{l}\text { Thr297(1HB) } \\
\text { Arg302(2HB })\end{array}$ & $\begin{array}{l}2.16 \\
1.96 \& \\
2.43\end{array}$ \\
\cline { 2 - 5 } & & & & 1.96 \\
& c-Myc & -7.2 & Tyr131(1HB) & 2.47 \\
& & Lys259(1HB) & 2.43 \\
\hline Afzelin & HIF-1 $\alpha$ & -6.3 & Lys350(1HB) & 2.31 \\
& & & Ala351(1HB) & 2.28 \\
\hline
\end{tabular}

When all selected phytocompounds docked into the binding pocket of $\beta$-catenin and $\mathrm{p} 53$, it was observed that both p53 and $\beta$-catenin have binding free energy $-8.8 \mathrm{kcal} / \mathrm{mol}$ and $-7 \mathrm{kcal} / \mathrm{mol}$ respectively for Bromelain. The binding pocket of both $\mathrm{p} 53$ and $\beta$-catenin are showing hydrogen bonding at Tyr275(1HB), Arg354(1HB), Asp316(1HB), Gln526(1HB), Arg477(2HB) and Gln274(1HB) and bond length is variable in the range of $1.99 \mathrm{~A}^{0}$ to $2.78 \mathrm{~A}^{0}$. (Fig.-4), Glu224 (1HB), Ser227(1HB), His $233(1 \mathrm{HB}), \mathrm{HOH} 2196(2 \mathrm{HB})$ the bond distance $1.85 \mathrm{~A}^{0}$ to $2.67 \mathrm{~A}^{0}$ (Fig.-3) respectively.

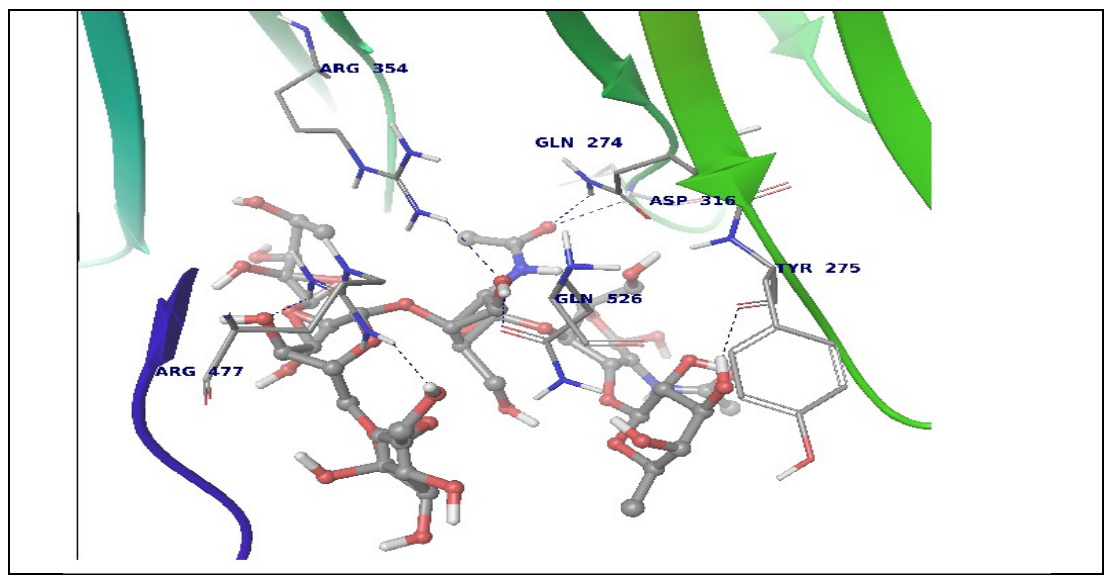

Fig.-3: Binding Mode of Lead Compound Bromelain with the Dynamically Stable Conformation of B-Catenin and Major Binding Mode of Bromelain are the Blue Dotted Line Indicating Hydrogen Bonds.

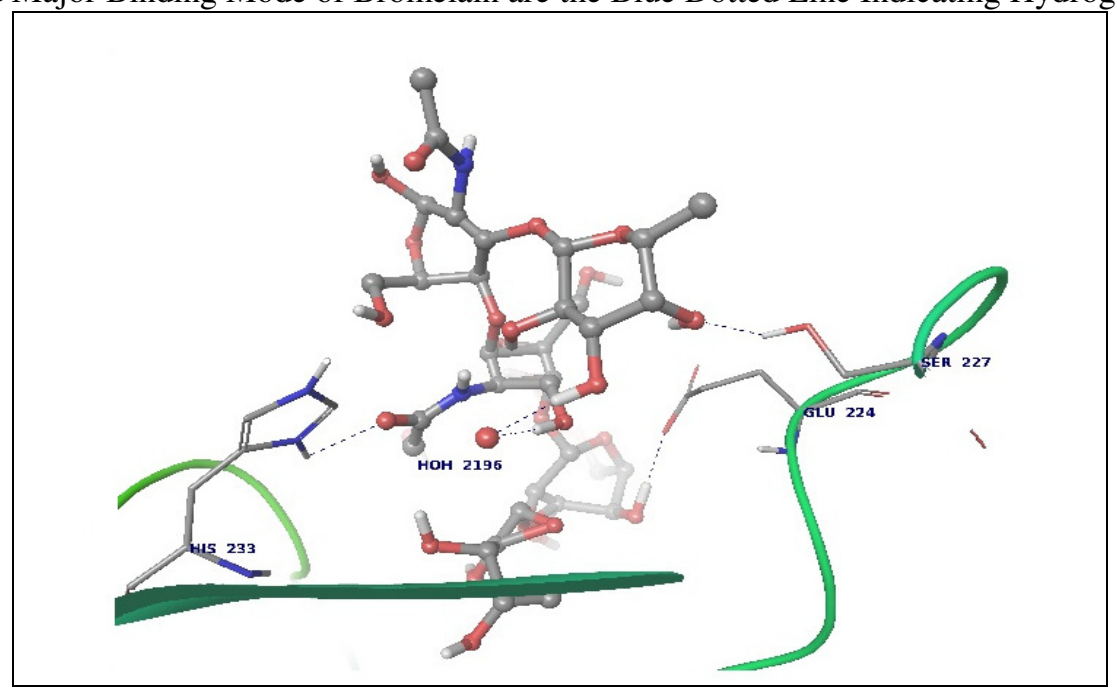

Fig.-4: Binding Mode of Lead Compound Bromelain with the Dynamically Stable Conformation of P53 and Major Binding Mode of Bromelain are the Blue Dotted Line Indicating Hydrogen Bonds

Similarly, another phytocompound Harpagoside was docked to AP-1 and c-Myc, after docking Harpagoside was showing ideal binding free energy and RMSD as compared to other compounds (Fig.- 
5 and 6). When Harpagoside was docked AP-1 and c-Myc, resulting binding free energy was -6.0 $\mathrm{kcal} / \mathrm{mol},-7.2 \mathrm{kcal} / \mathrm{mol}$ respectively. The binding pocket of AP-1 was made up of Thr297 (1HB) and Arg302 (2HB), there was a hydrogen bonding between these amino acids and the ligand and bond length was in the range of $1.96 A^{0}-2.43 A^{0}$ (Fig.-7).

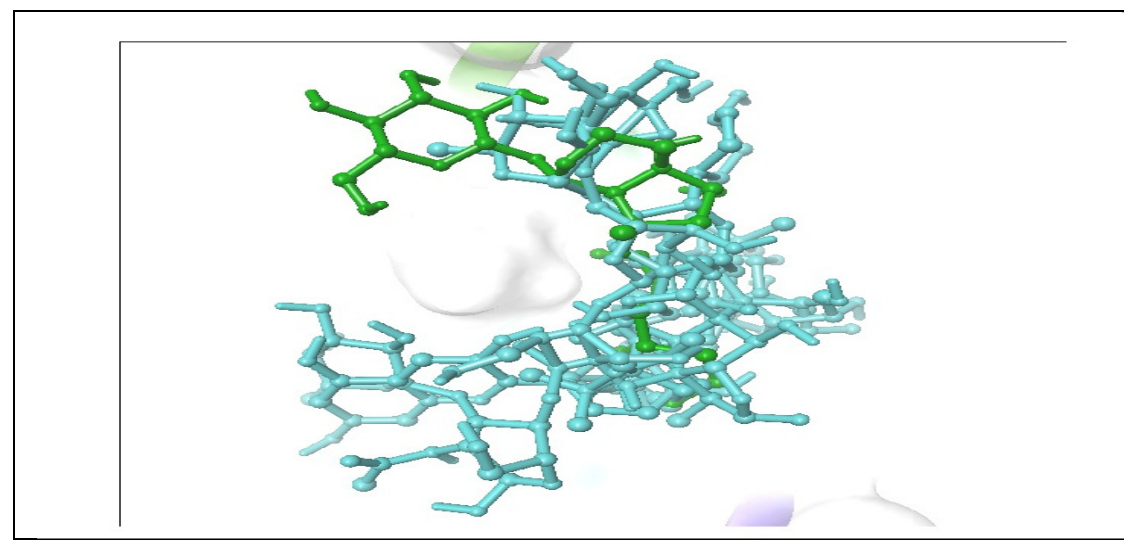

Fig.-5: Superposition of the Docking Poses of Ten Phytocompounds in Active Site Of Dynamically Stable Structure Of AP-1. The Structure of all Phytocompounds is Represented in the Blue Color and the High Affinity Compound Harpagoside Represented in The Green Color.

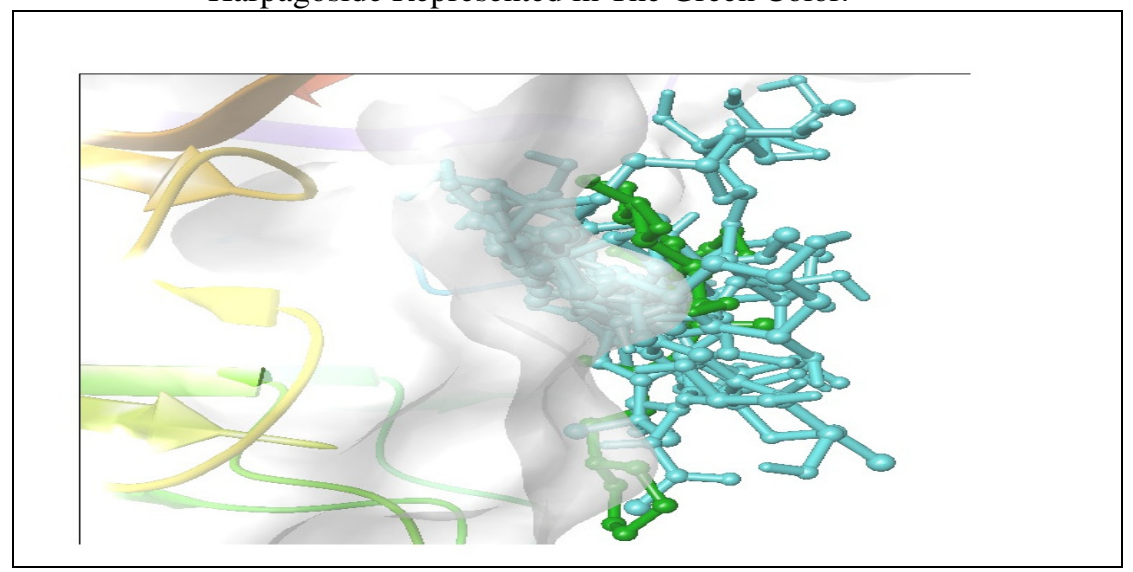

Fig.-6: Superposition of the Docking Poses of Ten Phytocompounds in Active Site of Dynamically Stable Structure Of C-Myc. The Structure of all Phytocompounds is Represented in the Blue Color and The High Affinity Compound Harpagoside Represented in the Green Color.

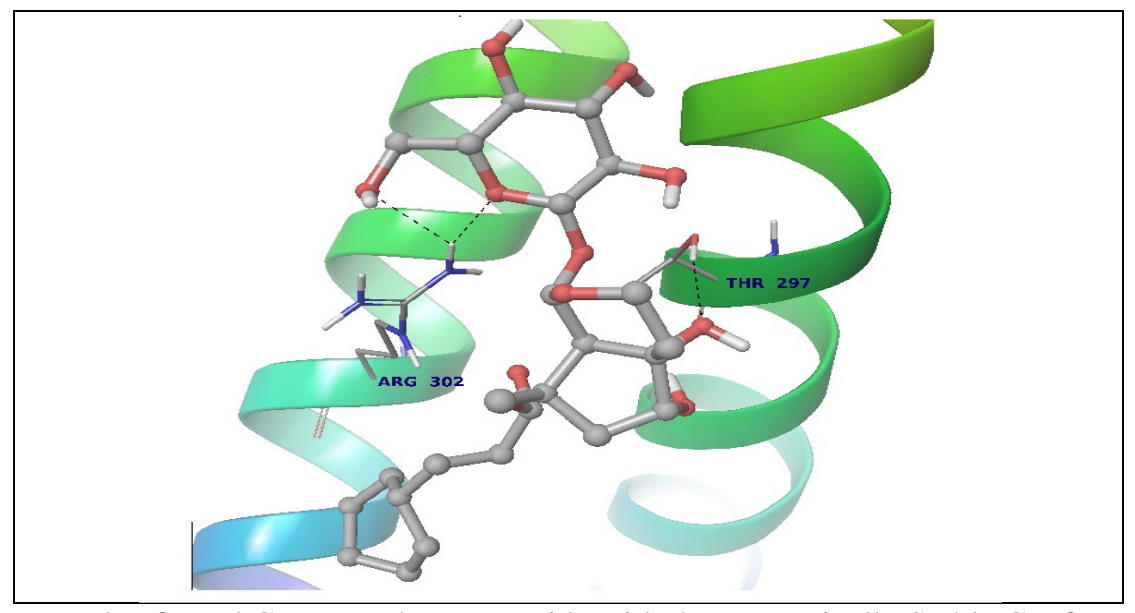

Fig.-7: Binding Mode of Lead Compound Harpagoside with the Dynamically Stable Conformation of AP-1 and Major Binding Mode of Harpagoside are the Yellow Dotted Line Indicating Hydrogen Bonds. 
Similarly binding pocket of c-Myc was made up of Tyr131 (1HB), Lys259 (1HB) and HOH646 (1HB) and hydrogen bonding between amino acids and atoms of a ligand with the bond length $1.96 \mathrm{~A}^{0}-2.47 \mathrm{~A}^{0}$ (Fig.-8). Eventually, it is reported that the third compound Afzelin as a lead compound for HIF-1 $\alpha$ showed a binding energy of $-6.3 \mathrm{kcal} / \mathrm{mol}$ when compared with other compounds (Fig.-9) and it was indicating hydrogen bonding between amino acids Lys350(1HB) and Ala351(1HB) and atoms of ligand with bond length $2.28 \mathrm{~A}^{0}$ and $2.31 \mathrm{~A}^{0}$ respectively (Fig.-10).

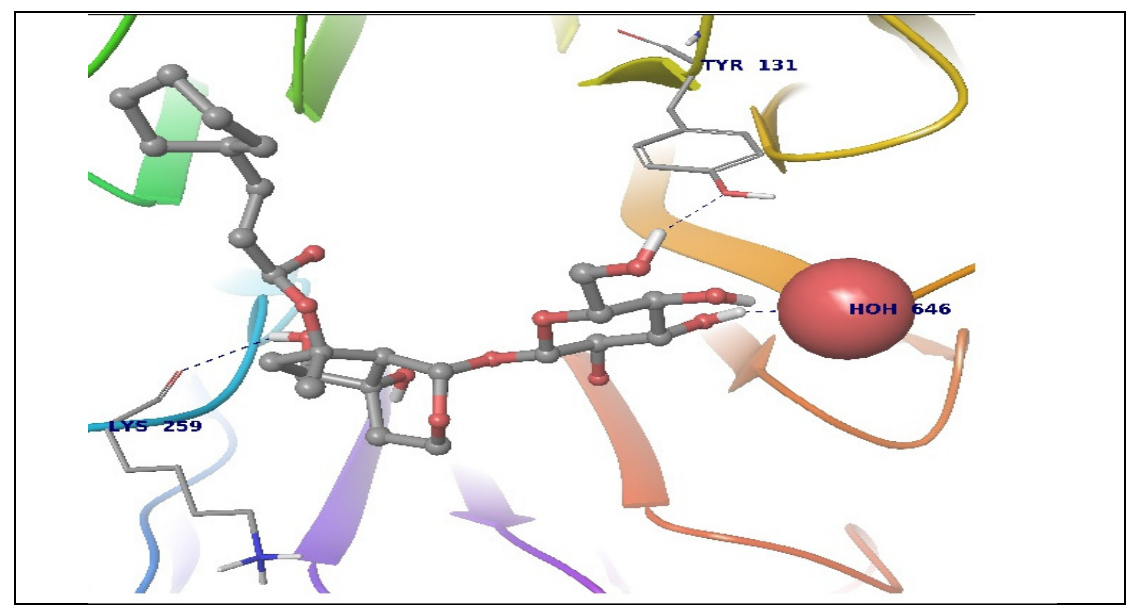

Fig.-8: Binding Mode of Lead Compound Harpagoside with the Dynamically Stable Conformation of C-Myc and Major Binding Mode of Harpagoside, are the Blue Dotted Line Indicating Hydrogen Bonds.

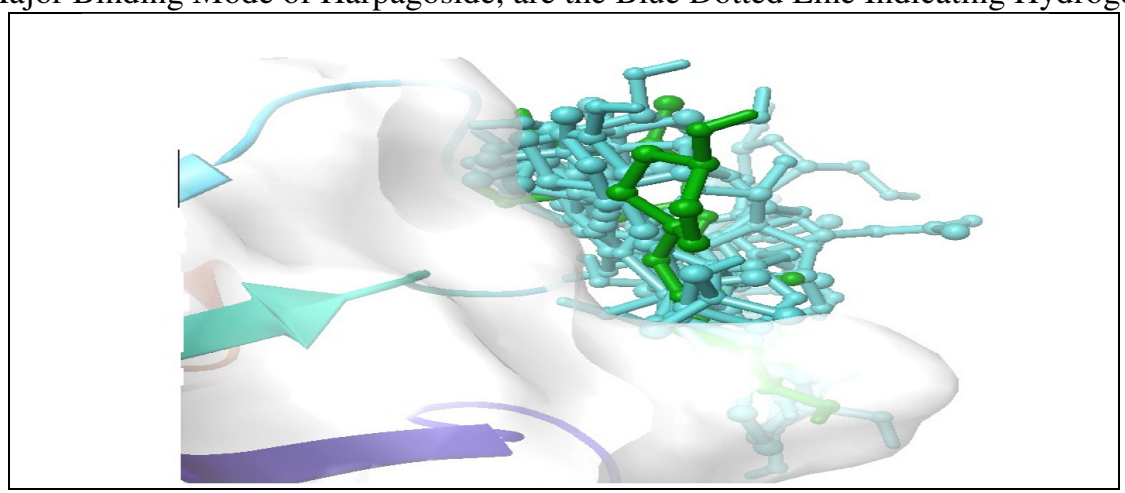

Fig.-9: Superposition of the Docking Poses of Ten Phytocompounds in Active Site of Dynamically Stable Structure of HIF-1 $\alpha$. The Structure of all Phytocompounds is represented in the Blue Color and the High Affinity Compound Afzelin Represented in the Green Color.

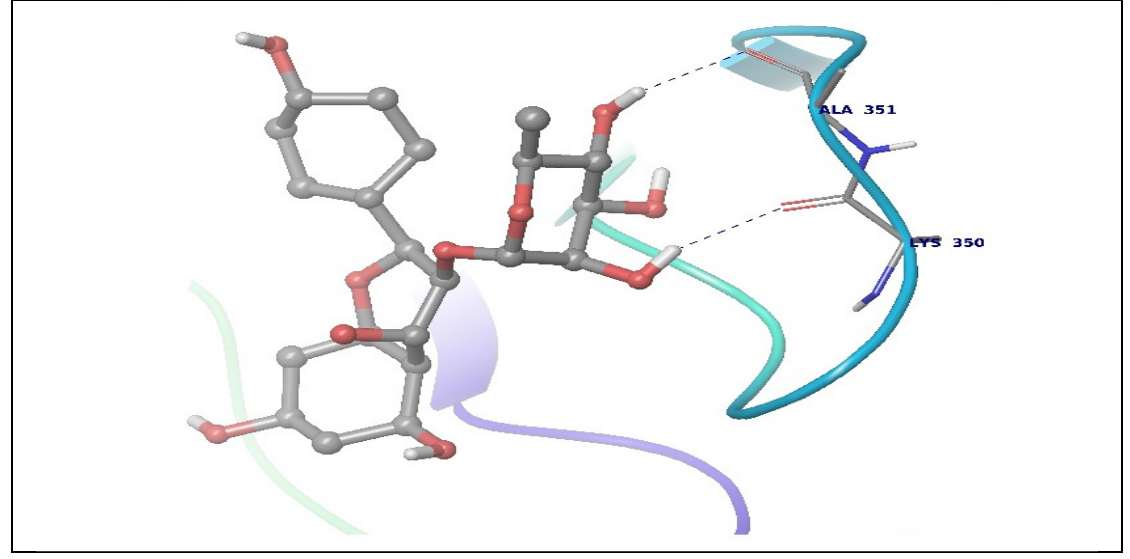

Fig.-10: Binding mode of lead compound Afzelin with the dynamically stable conformation of HIF- $1 \alpha$ and major binding mode of Afzelin, the blue dotted line indicates hydrogen bonds. 


\section{CONCLUSION}

After a thorough analysis of result section, it was observed that Bromelain, effectively binds to transcription factor $\mathrm{p} 53$ and $\beta$-catenin, which was evident from the binding free energy and RMSD values after docking. During docking, atoms in the pharmacophore region of Bromelain and p53 \& $\beta$ catenin are not deviating much from each other; it shows that there was strong affinity of Bromelain towards p53 and $\beta$-catenin. Based on docking score, the study can be concluded reporting that the compound Bromelain is more effective against $\beta$-catenin and p53 as compared to other transcription factors (fig.1, 2). Based on this observation, Bromelain can be used as effective drug in Hepatocellular carcinoma (HCC). The second lead being Harpagoside compound for AP-1 and c-Myc receptors and the third lead compound Afzelin, for HIF-1 $\alpha$. The most considerable validation method for evaluating the accuracy of docking procedure is by re-docking analysis.

The present study performed a computer-aided screening approach and concluded that these abovementioned lead compounds prove to be potential phytocompounds that can act as a lead therapeutic agent against $\mathrm{HCC}$ and further investigation is being done on its molecular mechanisms.

\section{ACKNOWLEDGMENT}

The authors are thankful for the support extended by Mr.Pawan Kumar Puvvada CEO and M.D, Sun Biologicals, Bangalore, Mr. Shivandappa, Associate Professor, RV Engineering College, Bangalore and Department of Chemical Engineering, JNTUA, Ananthapur for their kind cooperation in completing this research work successfully.

\section{REFERENCES}

1. T.I. Lee, and R.A. Young, Cell, 152, 1237(2013), DOI: 10.1016/j.cell.2013.02.01

2. H. Singh, A.A. Khan, and A.R Dinner, Trends Immunol., 35, 211(2014), DOI: 10.1016/j.it.2014.03.006

3. A.P. Fong and S.J. Tapscott, Current Opinion in Genetics and Development, 5(23),568 (2013), DOI: $10.1016 /$ j.gde.2013.05.002

4. G.Bejerano, M. Pheasant, I. Makunin, S. Stephen, W.J. Kent, J.S. Mattick and D. Haussler, Science 304,1321(2004)

5. Samuel A. Lambert, Arttu Jolma, Laura F. Campitelli, Pratyush K. Das, Yimeng Yin, Mihai Albu, Xiaoting Chen, Jussi Taipale, Timothy R. Hughes, and Matthew T. Weirauch, Cell ,172, 661(2018), DOI:10.1016/j.cell.2018.01.029

6. M.C. Kew, Journal of Hepatocellualar Carcinoma, 1, 115(2014), DOI:10.2147/JHC.S44381

7. A. Forner , J.M. Llovet and J. Bruix, The Lancet, 379, 1245(2012), DOI:10.1016/S01406736(11)61347-0.

8. R.H. Morse, Journal of Cellular Biochemistry, 105, 560(2007), DOI:10.1002/jcb.21493

9. A.H. Brivanlou and J.E. Darnell Jr, Science, 295, 813(2002), DOI:10.1126/science. 1066355

10. T. Juven-Gershon and J.T. Kadonaga, Developmental Biology, 339, 225(2010), DOI:10.1016/j.ydbio.2009.08.009

11. Pelengaris, M. Khan and G. Evan, Nature Review Cancer, 2, 764(2002), DOI:10.1038/nrc904

12. G. L. Kelly and A.B. Rickinson, Hematology Am. Soc. Hematol. Educ. Program, 84, 277(2007), DOI:10.1182/asheducation-2007.1.277

13. Y. Wei, JT. Van Nhieu, S. Prigent, P. Srivatanakul, P. Tiollais and MA. Buendia, Hepatology, 36, 692(2002), DOI: 10.1053/jhep.2002.35342

14. T. Suzuki, H. Yano, Y. Nakashima, O. Nakashima, M. Kojiro, Journal of Gastroenterol Hepatol, 17, 994(2002), DOI:10.1046/j.1440-1746.2002.02774.X

15. E.F.Wagner, Oncogene, 20, 2334(2001), DOI: 10.1038/sj.onc.1204416

16. W.S. el-Deiry, S.E. Kern and J.A. Pietenpol, Nature Genetics,1, (1992), DOI:10.1038/ng0492-45

17. J. Momand, G. P. Zambetti, D. C. Olson, Cell, 69, 1237(1992), DOI:10.1016/0092-8674(92)90644$\mathrm{r}$

18. M. Muller, S.Strand, Journal of Clinical Investigation, 99, 403(1997), DOI: 10.1172/JCI119174 
19. J.H. Abdel Kareem Azab ,P. Quang, F. Azab, C. Pitsillides, R. Awwad, B.Thompson, P. Maiso, J.D. Sun, C.P. Hart, A.M. Roccaro, A. Sacco, H.T. Ngo, C.P. Lin, A.L. Kung, R.D. Carrasco, K. Vanderkerken, I.M. Ghobrial, Blood, 119, 5782(2012), DOI: 10.1186/s13046-017-0533-1

20. https://pubchem.ncbi.nlm.nih.gov/

21. An elementary mathematical theory of classification and prediction, IBM Report, G. Salton, Automatic Information Organization and Retrieval. McGrawHill, 238, (1968).

22. C.A. Lipinski, Drug Discovery Today: Technologies, 1(4), 337(2004), DOI: 10.1016/j.ddtec.2004.11.007

23. Hanwell, Journal of Cheminformatics, 4, 17 (2012) , DOI:10.1186/1758-2946-4-17

24. http://www.rcsb.org/

25. G.M. Morris , R. Huey, W. Lindstrom ,M.F. Sanner , R.K. Belew , D.S. Goodsell , A.J. Olson, Journal of Computational Chemistry, 30, 2785(2009), DOI: 10.1002/jcc.21256

26. Trott, Oleg and J. Olson, Arthur, Journal of Computational Chemistry, 31,455(2009), DOI: $10.1002 / j$ jc. 21334

27. http://www.accelrys.com/

28. S. Ganguly, S. Murugesan, Rasayan Journal of Chemistry,1(2),251(2008)

[RJC-5475/2019] 